\title{
Catálogo de libros científicos enviados por Meerman a Mayans
}

\author{
ANTONIO MESTRE ***
}

Después de los trabajos de Vicente Peset y López Piñero ${ }^{1}$, no se puede defender el aislamiento científico español respecto a Europa hasta bien entrado el siglo XVIII. Otra cosa es la valoración de nuestras aportaciones. El descubrimiento de la aportación de los "novatores" ha constituido un hecho fundamental en el conocimiento de nuestro desarrollo científico. Porque, si al hecho concreto de las ciencias experimentales, añadimos la penetración de las ideas filosóficas (Quiroz Martínez ${ }^{2}$ ) y del humanismo a través de Martí (Mestre y Luis Gil), tenemos que pensar en una nueva visión del Siglo de las Luces en España.

No voy a insistir en ese aspecto, hoy ya generalmente reconocido. Limitaré mi aportación a un hecho concreto de comunicación intelectual en el campo científico entre Mayans y Gerardo Meerman. Ni el valenciano ni el holandés eran profesionales del estudio de las ciencias expe-

* Universidad de Valencia.

** Transcribo literalmente, tanto los nombres de los autores como los títulos, con la misma ortografía que aparece en el original.

1 V. PESET, “La Universidad de Valencia y la renovación científica española (16871727)", en Asclepio, XVI, 1964, 214-231; ID., Gregori Mayans i la cultura de la II.lustració, Barcelona-Valencia 1975; J. M. LOPEZ PIÑERO: La introducción de la ciencia moderna en España, Barcelona 1969; ID., Ciencia y técnica en la España de los siglos XVI y XVII, Barcelona 1979; V. NavarRo: Tradició i canvi cientific al Pais Valenciá modern (1660-1720), Valencia 1985.

${ }^{2}$ O. V. Quiroz Martínez: La introducción de la filosofía moderna en España, México 1949. 
rimentales. Su relación epistolar duró prácticamente 24 años. Iniciada en 1747, finaliza en 1771 con la muerte de Meerman.

El holandés gozaba de una situación económica privilegiada (sobrino de Juan de la Coste, presidente de la Compañía Holandesa de las Indias Orientales) y formaba parte de una familia de larga tradición política, pues entre sus parientes antepasados se incluía Hugo Grocio. Con una buena formación humanista y dedicado al estudio de la jurisprudencia, desde muy joven se hizo notar en el campo de las letras $y$, por medio de los editores lioneses hermanos Deville, entró en correspondencia literaria con Mayans.

Quisiera señalar que se trata de una verdadera intercomunicación. Si Meerman aporta sus conocimientos literarios o jurídicos - en este sentido, constituye una asombrosa apertura al mundo cultural germánicotambién Mayans ${ }^{3}$ expone sus extraordinarios conocimientos jurídicos 0 históricos. Es una comunicación amplia, sincera y enriquecedora para ambos corresponsales.

Es necesario insistir, sin embargo, en que la correspondencia no abarca todos los campos de la cultura con la misma intensidad. Resulta lógico que, tratándose de juristas, el aspecto mejor estudiado es, sin duda, la jurisprudencia. En este sentido, la aportación de Mayans fue realmente extraordinaria. Constituia su campo de estudio profesional y puso a disposición de Meerman no sólo los libros de la riquísima biblioteca sino hasta los manuscritos de que disponía. Sólo así hizo posible que Meerman incluyera en sus Novus thesaurus iuris civilis et canonici la serie de juristas españoles que influyeron decisivamente en la historia de la jurisprudencia: Ramos del Manzano, José de Retes, Altamirano, Nicolás Antonio, Quintanadueñas... y que constituyen la aportación más interesante de la obra de Meerman. Tanto es asi que el holandés dedicó el Novus thesaurus... a su amigo Gregorio Mayans, a cuyo entusiasmo debía tantas noticias ${ }^{4}$.

${ }^{3}$ A. Mestre: llustración y reforma de la Iglesia. Pensamiento político-religioso de don Gregorio Mayans y Siscar (1699-1781), Valencia 1968; ID., Historia, fueros y actitudes politicas. Mayans y la historiografía del XVIII, Valencia 1970; G. MAYANS y SISCAR: Epistolario II. Mayans y Martí, Valencia 1973; L. GIL: “El deán Martí o la esperanza fallida», en Tres grandes humanistas españoles, Madrid 1975; G. Malansius: E. Martini, decani alonensis, vita, Valencia 1977.

"A. MESTRE: “La difusión de la cultura española del XVIII en los Países Germánicos". Mayans y el círculo de Meerman", en Influjo europeo y herencia hispánica, Mayans y la ilustración valenciana, Valencia 1987. 
Un segundo campo de comunicación se centra en el humanismo. Es bien sabido que nunca hemos manifestado los españoles un excesivo entusiasmo por las humanidades. Mayans, que sí lo sentía, tuvo que recurrir en muchos casos a su amigo holandés para conseguir las mejores ediciones de los clásicos. No hay que olvidar, sin embargo, el envio que hace don Gregorio de las obras de nuestros humanistas del xVI, como Pedro Juan Núñez.

Finalmente, nuestra historia interesaba de manera especial a Meerman y a sus amigos holandeses y alemanes. En este sentido, su curiosidad no tiene límites y abarca desde la arqueología y epigrafía hasta los ensayos sobre las letras ibéricas (Velázquez) o los estudios de historia eclesiástica (Flórez).

En los tres aspectos indicados, la aportación mayansiana fue importante. En cambio, en el mundo de la ciencia experimental fue mucho menor: se limita a enviar los libros solicitados por Meerman. $Y$ puede fácilmente comprenderse que fueron pocos: las obras de Jorge Juan y Ulloa, de Quer y hasta el Tratado de la electricidad de Benito Navarro (1752)...

Tampoco sobresale, en líneas generales, la aportación de Meerman en el campo científico. Mayans no pedía muchos libros de ciencia experimental -estaba fuera de su campo de interés específico- ni hablaba con mucha frecuencia de semejantes temas. En cambio y desde el primer momento, el holandés había expresado su idea de que en el campo de las matemáticas y astronomía, preferia los autores anglosajones a los que concedía la supremacía. En esa línea manifestará, asimismo, su escaso aprecio de las aportaciones científicas de Feijoo.

Sin embargo, las cosas cambiaron en 1766. Mayans fue premiado por el Gobierno de Carlos III con el título de Alcalde de Casa y Corte y una pensión vitalicia. Y Manuel de Roda, que ya conocía la decisión de expulsar a los jesuitas y maquinaba la reforma de la enseñanza en las Universidades españolas, encargó a don Gregorio la redacción de un plan de estudios. Era una empresa de altos vuelos que el valenciano cumplió con dignididad. Hoy conocemos la aportación mayansiana en la ldea del nuevo método que se puede practicar en la enseñanza de las Universidades de España ${ }^{5}$.

${ }^{5}$ J. L. y M. PESET: Mayans y la reforma universitaria. Idea del nuevo método que se puede practicar en la enseñanza de las Universidades de España, Valencia 1975. 
Ahora bien, dada la amplitud de la empresa, don Gregorio midió sus fuerzas. En el campo de la Jurisprudencia y Humanidades su preparación era más que suficiente. También se consideraba con suficientes conocimientos para exponer los criterios reformistas en el estudio de Teología y Filosofía. Pero no se atrevió a enfrentarse con los textos relativos a las ciencias experimentales: Matemáticas, Física y Química, Medicina... Y recurrió a su amigo Meerman.

Tenemos la respuesta del holandés. La alegría por el premio concedido al valenciano es desbordante, hasta llamar al "rey católico, verdadero Musagetes", con el deseo de que disfrute de tales beneficios durante su larga vejez que desea alcance la edad de Néstor, siempre en favor del bien común. Respecto al encargo mayansiano sus palabras constituyen todo un síntoma: «Indicem optimorum librorum in Medicina ac Mathesi, studiosae iuventuti convenientium, habebis proxime; opus quippe ut et aliorum consilium hac in parte implorem. Necesse vero est, ut in scholis atque academiis vestris introducantur Metaphysica Leibnitiana, Philosophia Newtoniana, Medicina Hippocratico-Boerhaviana” 6 .

La carta de Meerman es del 2 de febrero de 1767 y sus palabras demuestran una postura claramente definida. Es cierto que no se atreve a señalar inmediatamente la bibliografía más importante. Tampoco Meerman era un científico y necesitaba consultar a los especialistas. Pero la línea resulta evidente: estudios filosóficos en la línea de Leibniz, la físicamatemática según Newton y la medicina de acuerdo con la escuela de Boerhaave.

Apenas habian transcurrido dos meses, concretamente el 4 de abril de 1767, Meerman enviaba el catálogo de libros científicos. Se trata, en su mayoria, de textos latinos y franceses y el holandés se atreve a aconsejar los libreros más adecuados. Para los libros latinos recomienda a los hermanos Luchtmans de Leyden, mientras que para los franceses aconseja a Juan Miguel Rey (Amsterdam) y a Pedro Gosse (La Haya).

No voy a repetir el catálogo que transcribo íntegro con los títulos, grafía y año de edición como los envió Meerman. Sólo quisiera señalar que están todas las figuras de primera línea en cualquier campo científico: Newton, Euler, D'Alembert, Haller, Boerhaave, Linneo, Muschen-

\footnotetext{
${ }^{6}$ BMV, Serrano Morales 6810-1.
} 
broeck, Wolf... La información es exhaustiva y alcanza a figuras que, sin la importancia y el renombre de los anteriores, significaban las aportaciones más recientes: la Optica de Bouguer (1762) o la traducción del tratado de Smith (1766), los tratados astronómicos de La Lande (1762 y 1764), la Astronomía del P. Pezenas (1766), las obras astronómicas de Duverney (1761) o las Instituciones pathologiae de T. G. Ludwig así como su Methodus doctrinae medicae universae (1766)...

No era la primera vez que aparecían entre nosotros algunos de los nombres más significativos. Tampoco será la última. Líneo y, sobre todo, Haller fueron corresponsales de Antonio Capdevila (amigo-discípulo de Mayans) que facilitó al médico suizo datos publicados en su Bibliotheca medica. Hoffman era citado por Mayans en 1754; Gerardo van Swieten era conocido en Valencia en 1753 así como Bellini y Juan Gorter eran citados junto con Boerhaave, Haller y Swieten en $1760^{7}$. La penetración de tales autores y otros no menos caracterizados debió ser intensa en Valencia. Así lo demuestra el estudio de Florensa basado en los memoriales de opositores a cátedras ${ }^{8}$. Asimismo, Boerhaave, Muschenbroeck y Bellini eran propuestos como libros de texto en el plan de estudios presentado por el Claustro de la Universidad de Salamanca en $1766^{9}$. Más tarde, Boerhaave, Lorenzo Heister y Gorter fueron propuestos en el plan de estudios de Olavide (1769) y de nuevo Boerhaave, con Macquer, Gorter, Beaume, Tesari y Home, aparecerán en el plan Blasco (1786) ${ }^{10}$. Y cualquier especialista podría multiplicar las citas. Pero en conjunto creo que el catálogo de libros enviado por Meerman constituye un programa muy completo en el campo de la enseñanza de la ciencia experimental en la España del XVIII.

Tampoco pretendo sacar todas las conclusiones que los historiadores de la medicina y de la ciencia deducirán con mejor conocimiento. Pero sí quisiera insinuar la línea de penetración que supone el catálogo. Resulta lógica la importancia que concede a la ciencia holandesa-alemana. La Universidad de Leyden ocupa, por supuesto, un papel prepon-

7 G. MAYANS y Siscar: Epistolario I, Mayans y los médicos, Valencia 1972.

${ }^{8} \mathrm{~J}$. FLORENSA, "Filosofía en la Universidad de Valencia (1733-1787) según los opositores a cátedra de filosofía", en Analecta calasanctiana, 21 (1969) 99-212.

9 J. L. PESET: "Andrés Piquer y la enseñanza de la medicina", en / Congreso de Historia del Pais Valenciano, Valencia 1971, III, 725-729.

${ }^{10} \mathrm{~F}$. Aguilar Piñal: Plan de estudios para la Universidad de Sevilla, Barcelona 1969; Plan de estudios aprobado por S. M. y mandado observar en la Universidad de Valencia, Valencia 1787. 
derante. Desde Boerhaave parte una serie de médicos (Haller, Swieten...) que extendieron su método en Suiza y Alemania central (Lausana, Leipzig, Gotinga...) y que llegaron, en sus manifestaciones cientificas, a Petrogrado por medio de Euler y los Bermouille. El testimonio de la bibliografía facilitada por Meerman no deja lugar a dudas.

Ahora bien, como no podía ser menos, la ciencia anglosajona está presente. Newton en primer lugar, pero también Gregory, Smith, Flamsteed... Finalmente, las frecuentes alusiones a París (D'Alembert, La Caille, Bouguer, Le Bond...) y las obligadas a ciertos científicos italianos (Albini, por ejemplo) demuestran una amplitud de conocimientos nada común.

Uno piensa instintivamente qué hubiera ocurrido si estos textos científicos se hubieran impuesto y explicado en las Universidades españolas a fines del XVIII. Quizás el ambiente y la misma preparación intelectual del profesorado no lo admitieran. Eso parece deducirse del hecho de que Mayans ni siquiera incluyera tales nombres en el plan de estudio que envió a Roda. Más todavia, tampoco en el Plan Blasco - redactado 20 años más tarde - puede verse un proyecto tan logrado en el campo de las ciencias experimentales. Pero ahí queda constancia de las noticias que llegaron a manos de Mayans en 1767 en un intento de adecuar nuestros estudios científicos a la investigación más avanzada en la Europa de las Luces.

\title{
CATÁLOGO DE LIBROS CIENTÍFICOS ENVIADO POR G. MEERMAN A G. MAYANS EL 4 DE ABRIL DE 1767
}

\begin{abstract}
G. J. S. Gravesande: Physices Elementa mathematica experimentis confirmata sive introductio ad Philosophiam Newtonianam, $4 .^{\circ}, 2$ vol. L(ugduni) B(atavorum), 1748.
\end{abstract}

\section{MECHANICA ET STATICA}

Traité de Dynamique par Monsr. D'AlemBert, 4. ${ }^{\circ}$, Paris 1743.

C. Hugonil, Opera mechanica, etc., illustrata a G. J. S. Gravesande, 4., 4 vols. L. B. 1751. 
Mecanique par Mr. de LA CAille, $8 .^{\circ}$

Scientia motus EULERI, Petrop. $4 .^{\circ}$

Mecanique de Mr. Trabaud.

\section{HYDRAULICA ET HYDROSTATICA}

D. Bernoulli: Hydrodynamica, $4 .^{\circ}$, Argentor. 1738.

De la resistance des fluides par Mr. D'AlEMBERT, 4. ${ }^{\circ}$, Paris 1752.

Traité des Fluides par Mr. D'Alembert, 4. ${ }^{\circ}$, Paris 1744.

POleni: De motu aquarum mixto, Patav.

GuLlielmi: De cursu fluviorum, Genèv.

Architecture hydraulique, par (Forest de) Belidor, $4 .^{\circ}, 4$ vol. Paris 1750.

\section{ÓPTICA}

LA CAILle: Leçons d'optique, 8. ${ }^{\circ}$, Paris 1756.

Optica, auctore I. Newton, latine reddita a I. Clarke, $4 .^{\circ}$, Laus. et Genev. 1740.

Optique par Mr. Bouguer, 4., Paris 1762.

Traité d'optique par Mr. SMITH, traduit de l'anglais, 4. ${ }^{\circ}$, Breslaf. 1766.

MÚSICA

Tentamen theoriae musicae, auctore EULERO, $4 .^{\circ}, 1739$.

Traité de musique par Mr. D'AlemBert, $8 .^{\circ}$, Paris. 


\section{ASTRONOMÍA}

Leçons elementaires d'Astronomie par Mr. de LA CAILLE, 8. ${ }^{\circ}$, Paris 1751. Exposition du calcul astronomique par Mr. de LA LANDE, 8. ${ }^{\circ}$, Paris 1762. Astronomie par Mr. de LA LANDE, 4. ${ }^{\circ}, 2$ vol. 1764.

Institutions astronomiques, $4 .^{\circ}$, Paris 1746 , par MONNIER.

D. Gregoril: Astronomiae Elementa, 4. ${ }^{\circ}, 2$ vol. Genèv. 1726.

Connoissances des temps par Mr. de LA LANDE, Paris $8 .^{\circ}$, quovis anno prodit.

Tabulae solares, auctore de LA CAILLE.

Tabulae lunares MAYERI. Vide Astronomie de LA CAILLE.

Flamstedil, Historia et Atlas coelestis Britannica, fol. Lond. 1725, 4 vol.

\section{SCIENTIA NAVALIS}

EULERI: Scientia navalis, $4 .^{\circ}, 2$ vol., Petropol. 1749.

Traité du navire, de sa construction et de ses mouvements, par Mr. BouGUER, $4 .^{\circ}$, Paris 1746.

Du HAMEL: Architecture navale, 4. ${ }^{\circ}$, Paris 1752.

Astronomie des marins par le pére PezenAS, Paris $1766,8 .^{\circ}$

De la manoeuvre des vaisseaux ou traité de Mecanique et de Dinamique, par Mr. BOUguen, 4. ${ }^{\circ}$, Paris 1757.

Traité du Pilotage, par Mr. VILLehuet, Paris 1756. 


\section{PYROTECHNICA ET ARS MILITARIS}

Essai d'une theorie d'artillerie, par Mr. d'ARCY.

BELlDOR: Traité d'artillerie, avec la science des ingenieurs et les fortifications, $4 .^{\circ}$, Paris 1739.

LE BLOND: Elemens de fortification, 8. ${ }^{\circ}$, Paris 1764.

Constitutions militaires, avec une Tactique, 4., Paris 1760.

L'esprit de Frolard sur Polybe, 8. , Lyon 1761.

Esprit des loix de tactique par BonneVILLe, 4.․, LA HAYE, 2 vol. 1762.

\section{METAPHYSICA ET LOGICA}

G. J. S. Gravesande: Introductio ad Philosophiam, 8. ${ }^{\circ}$, Leid. 1756.

F. C. BAUMEISTER: Institutiones metaphysicae, Vitemberg. 8. ${ }^{\circ}, 1762$.

F. C. BaumeIster: Institutiones Philosophiae rationalis, $8 .^{\circ}$, Vitemberg. 1759.

\section{ANATOMÍA}

L. HeYSTERI: Compendium anatomicum, 8., 2 vols. Genèv. 1748.

Anatomie chirugicale de J. PALFIN, refondue par H. PETIT, 8., 2 vol. Paris 1763.

Essais anatomiques avec la maniere de dissequer, par LIEUTAUD, $8 .^{\circ}$, Paris 1766.

J. B. Winslow: Exposition anatomique de le structure du corp humain, Paris 1732.

Oevres anatomiques de Mr. Duverney, 4. ${ }^{\circ}, 2$ vol. Paris 1761.

Icones ossium foetus, B. S. ALBINI, 4. , L. B. 1737. 


\section{PHYSIOLOGIA}

A. V. HALler: Primae lineae Physiologiae, $8^{\circ}$, Gotting. 1765.

G. E. Hambergeri: Elementa Physiologiae medicae, 8. ${ }^{\circ}$, lenae 1757.

H. Boerhaven: Praelectiones academicae, editae et notis illustratae ab A. HALlero, 8., 7 vol. Gotting, 1745.

Elementa Phisiologiae, C.H., auctore A. V. HALLeR, 4. ${ }^{\circ}, 8$ tom.

\section{PATHOLOGIA}

M. D. GaUBII: Institutiones pathologiae medicinalis, 8. ${ }^{\circ}$, Leida 1763.

C. G. LuDWIG: Institutiones Pathologiae, Lipsiae 1754.

-: Therapeutica et praxis medica.

H. Boerhaven: Aphorismi de cognoscendis et curandis morbis, $8^{\circ}, 1737$.

J. DE Gorter: Praxis medicae systhema, $8 .^{\circ}, 2$ vol. Harderovici 1750 et Lipsiae, 4. ${ }^{\circ}, 1755$.

OOSTERDYK SCHACHT: Institutiones medicinae practicae, $8 .^{\circ}$, Ultraject. 1747.

C. G. LUDWIG: Methodus doctrinae medicae universae, 8. ${ }^{\circ}$, Lipsiae 1766.

C. G. LuDWIG: Institutiones therapiae generalis, $8^{\circ}{ }^{\circ}$, Lipsiae 1754.

G. VAN ZWIETEN: Commentaii in H. Boerhaven aphorismos de cognoscendis et curandis morbis, $4 .^{\circ}, 5$ tom. 1752.

A. DE HAEN: Ratio medendi, $8 .^{\circ}, 11$ tom. Viennae.

F. Hormanni: Opera omnia physiologica medica, fol. 6 vol., et suplementum, 3 vol. Genev. 1740. 
J. B. MORGANI: De sedibus et causis morborum per anatomicos indagatis, fol.

\section{MATERIES MEDICA}

Cynosura materiae medicae a JoHA. BeCClero, $4 .^{\circ}$, Argentor. 1745,3 vol.

R. A. Vogel: Historia materiae medicae, Lugduni Batavor. et Lipsiae 8. 1758, Frank 1766.

H. Boerhaven: De materia medica, L.B. $1762,8 .^{\circ}$.

H. D. GauBII: Libellus de methodo concinandi formulas medicinales, $8 .^{\circ}$, L.B. 1752 et 1766.

P. A. Parenty: De dosibus medicamentorum liber cum praefatione, H. D. GaUBII, 8. ${ }^{\circ}$, L.B. 1752 et 1761.

J. DE GORTER: Formulae medicinales cum indice virium, $8 .^{\circ}$, Amsteled 1755, Lipsiae 1760.

\section{CHEMIA ET PHARMACIA}

J. A. Crameri: Elementa artis docymasticae, L.B. $1744,8 .^{\circ}, 2$ vol.

R. A. Vogel: Institutiones Chemiae, L.B. et Lipsiae $1757,8 .^{\circ}$.

J. R. SPIELman: Institutiones Chemiae, Argentor 1766, $8 .^{\circ}$.

Elemens de Chymie par Mr. Macquen, 8. , 3 vol. Paris 1756.

H. Boerhaven: Elementa Chemiae, $4 .^{\circ}, 2$ tom. L.B.

Elemens de Chymie par Mr. de Moduy, $8 .^{\circ}, 6$ tom. Paris. 


\section{BOTANICE}

LINNOEl: Philosophia Botanica, $8 .^{\circ}$, Stookh, 1751, Vienn. 1765; Systhema naturae, $8 .^{\circ}$, Lypsiae 1748; Bibliotheca botanica, $8 .^{\circ}$; Species plantarum, $8 .^{\circ}, 7$ vol.

A. vON ROYEN: Prodromus hortus leidensis.

Familles des plantes par Mn. Adanson, 8. , 2 vol. Paris 1763.

C. G. LuDWIG: Institutiones historico-physicae regni vegetabilis, $8 .^{\circ}$, Lips. 1759.

C. G. LUDWIG: Definitiones generum plantarum, Lipsiae $1747,8^{\circ}$.

\section{MEDICINA FORENSIS}

J. BoHNIUS: De renuntiatione vulnerum.

C. G. LUDWIG: Institutiones medicinae forensis, Lipsiae 1761.

J. E. Hebenstreit: Anthropologia forensis, 8. ${ }^{\circ}$, Lipsiae 1753.

\section{CHIRURGIA}

G. D. Coschutz: Manuductio ad Chirurgiam rationalem, Brynsvigae 1755.

$4 .^{\circ}$.

J. DE GORTER: Chirurgia repurgata, 4. ${ }^{\circ}$, L.B. 1742.

Principes de Chirurgie par G. de la Faye, $8 .^{\circ}$, Paris.

Le chirurgien d'Hospital, par Mr. Belloste, $8 .^{\circ}, 2$ tom. Paris.

L. HeISTERI: Institutiones chirurgiae, $4 .^{\circ}, 2$ tom. 1750, Amstelad.

C. G. LUDWIG: Institutiones chirurgicae, Lipsiae, $1764,8 .^{\circ}$. 
J. Platnerl: Institutiones chirurgicae, Lipsiae, 8. ${ }^{\circ}, 1758$.

Traité des operations de chirurgie par H. F. LE Dran, Paris.

Cours d'operations par Mr. DIONIS, augmenté de remarques par G. de LA FAYE, 8. ${ }^{\circ}$, Paris 1757.

Traité des operations de Chirurgie, par R. J. C. de GARENGEOT, $8 .^{\circ}, 3$ vol. Paris.

Nouveau traité des instrumens de chirurgie par R. J. C. de GARENGEOT, $8^{\circ}, 2$ vol.

\section{EMBRYULCIA}

J. G. Roederer: Elementa artis obstetriciae, 8. , Gotting. 1759.

A. LeVRET: L'art des accouchemens, avec les observations de Suste, 8.', Paris.

SMELLIE: Traité des accouchemens, traduit de l'anglais, 8., 3 tom. Paris.

Puzos: Traité des accouchemens, $4 .^{\circ}$ Paris 1759.

H. Van Deventer: Ars obstetricandi, $44^{\circ}$.

F. Mauriceau: Traité des maladies des femmes grosses, 2 vol. $4 .^{\circ}$.

Traité des maladies des femmes grosses par J. Astruc, $8 .^{\circ}, 6$ tom. Paris 1761.

\section{TABULAE ANATOMICAE}

B. S. ALBINI, Tabulae scheleti humani, ossium, musculorum, uteri gravidi, ductus thoracici. 
B. Eustachil, Tabularum anatomicarum explicatio a B. S. ALBino, L. B. fol. 1744.

Iconum anatomicarum fasciculi, auctore A. VON HALLER, Gotting. 1743.

\section{TABULAE EMBRYULCAE}

Icones uteri humani, J. G. RODERER, fol. Gotting 1759.

\section{ARITHMETICA}

Arithmetica universalis, auctore Is. NEWTON.

Institutiones arithmeticas, PAULINI A S IOSEPHO, 4. ${ }^{\circ}$, Neapoli 1755.

Arithmeticae theoria et praxis a ANDREA TACQUET, 8..$^{\circ}$ Amstelod, 1704.

Arithmetica logarithmica, aucta por A. VLACQ, Goudae 1728.

Sherwin's Mathematical tables, by W. GardinER, London $8 .^{\circ}$.

\section{ÁLGEBRA}

Algebra, auctore RicaTI, Venet.

Traité d'Algebre, traduit de l'anglais de Monsr. Maclaurin, $4 .^{\circ}$, Paris 1753.

Leçons elementaires de mathematiques par Mr. de LA CAILLE, $8 .^{\circ}$, Paris 1756.

Elemens d'Algebre, par Mr. ClaiRANT, 8. ${ }^{\circ}$, Paris 1746.

Analyse demontrée par le P. Reyneau, $4 .^{\circ}, 2$ tom. 1756, Paris. 
Institutiones analyticae, auctore P. SAN IOSEPHO, 4. ${ }^{\circ}$, Neapoli 1735.

\author{
GEOMETRÍA
}

Euclidis elementa, cura SIMPsonIs, $4 .^{\circ}$, Glasg. 1756.

Euclidis elementa, opera BorrowII, $8 .^{\circ}$, Lond.

Geometrie par Mr. SIMPSON.

A. TACQET, Elementa euclidea geometriae planae ac solidae et selecta ex Archimede theoremata eiusdemque Trigonometria plane illustrata a G. WHISTON; accedit Trigonometria sphoerica R. J. BoscowisCH et Sectiones conicae G. Grand, Romae 1745, 8..$^{\circ} 2$ vol.

Euclidis quae supersunt omnia, ex recensione D. GREGORII, graeco-latina, Oxoniae 1703, fol.

Commentaires sur la geometrie de Mr. Descartes par C. RABUeL, $4 .{ }^{\circ}$, Lyon 1740.

Elemens de Geometrie par Mr. Clairant, 8. ${ }^{\circ}$ Paris 1752.

\title{
SECTIONES CONICAE
}

Sectiones conicae, auctore SIMPSON, Edimb. 4.

Sectionum conicarum elementa, auctore L. Trevigar, $4 .^{\circ}$, Cantabrig. 1731.

Traité des sections coniques par le marquis de L'HOPITAL, $44^{\circ}$, Paris 1707. 


\section{CALCULUS FLUSIONALIS}

Analyse des infiniment petits, par le marquis de L'HOPITAL, $4 .^{\circ}$, Paris 1706, avec le suitte par STONE 1735, 2 vol. $4 .^{\circ}$, Paris, et les aclairriseemens par Mr. VARIGNON, $4 .^{\circ}$, Paris 1720.

EULERI: Introductio in analysin infinitorum, $4 .^{\circ}$, Lausann. 1748, 2 vol.

—: Institutiones calculi differentialis, $4 .^{\circ}$, Petropoli 1755.

Bougainville: Calcul integral, 2 vol., Paris 1754.

\section{MATHESIS}

Philosophiae naturalis principia mathematica, auctore I. NEWTONIO, commentariis illustrata studio TH. LE SUEUR et FR. JACQUIER, $4 .^{\circ}, 3$ vol., Genev. 1739.

I. Newtoni: Opuscula collecta a JOH. Castillioneo, $4 .{ }^{\circ}, 3$ vol. Lausan et Genev. 1744.

G. J. S. Gravesande: Matheseos universalis elementa, 8. ${ }^{\circ}$, L. B. 1727.

J. A. Segneri: Cursus mathematicus, $8 .^{\circ}, 4$ tom. Hal. Magdeb. 1756.

C. WOLFII: Elementa matheseos universae, 4. ${ }^{\circ}, 5$ vol. Genev. 1743.

Elementa matheseos, auctore KARSTEN, Lipsiae $8 .^{\circ}$.

C. Maclaurin: Expositio philosophiae newtonianae, $4 .^{\circ}$, Vindob. 1761. 
Catálogo de libros científicos enviados por Meerman a Mayans

\section{PHYSICA}

P. Van Musschenbrock: Compendium Physicae experimentalis, $8 .^{\circ}, 2$ vol. L. B. 1762.

G. V. VIRAfFt: Praelectiones in Physicam theoreticam, 8. ${ }^{\circ}, 3$ vol., 1761.

G. J. S. Gravesande: Philosophiae newtonianae institutiones, $8 .^{\circ}$.

P. Van Musschenbroek: Introductio ad philosophiam naturalem, $4 . .^{\circ}, 2$ vol. L. B. 1762. 